\title{
Effet de la dose d'acide alpha-linolénique alimentaire sur le métabolisme lipidique
}

\author{
Anne MORISE ${ }^{1}$ \\ Dominique HERMIER $^{7}$ \\ Nicole COMBE ${ }^{2}$ \\ Philippe LEGRAND ${ }^{3}$ \\ Jacques MOUROT ${ }^{4}$ \\ Evelyne FENART ${ }^{5}$ \\ Pierre WEILL ${ }^{6}$ \\ ${ }^{1}$ Laboratoire de Physiologie de la Nutrition, \\ Université Paris Sud, 91405 Orsay \\ <anne.morise@rennes.inra.fr> \\ ${ }^{2}$ Iterg-Nutrition, 35405 Talence \\ ${ }^{3}$ Laboratoire de Biochimie de l'ENSAR, \\ 35000 Rennes \\ ${ }^{4}$ Senah, Saint-Gilles, \\ 35590 Saint-Gilles \\ ${ }^{5}$ Onidol, 75008 Paris \\ ${ }^{6}$ La Messayais, 35210 Combourtillé \\ ${ }^{7}$ UMR Physiologie de la Nutrition \\ et du Comportement alimentaire, \\ INAPG, \\ Paris, France
}

Les acides gras polyinsaturés (AGPI) de la famille $n-3$, qu'ils soient sous forme de précurseur (acide $\alpha$-linolénique, ALA) ou de dérivés (acides eicosapentaenoïque, EPA et docosahexaenoïque, DHA), sont connus pour leurs propriétés protectrices de différentes pathologies, notamment des maladies cardiovasculaires (MCV) [1-3]. Cependant la consommation actuelle de ces acides gras dans les pays occidentaux est bien inférieure aux recommandations [4-6] et doit être augmentée. Quelques huiles végétales riches en ALA et facilement disponibles, telles que les huiles de colza, de noix et dans certains pays, I'huile de lin, pourraient faciliter le suivi des recommandations. Toutefois, la teneur très élevée d'ALA de l'huile de lin pourrait entraîner des consommations d'ALA supérieures aux recommandations. II $s^{\prime}$ avère alors nécessaire de $s^{\prime}$ interroger sur les bénéfices d'une consommation importante de cet acide gras. En effet des études chez I'homme laissent penser qu'il pourrait y avoir une diminution de la conversion de l'ALA en ses dérivés à longue chaîne, lorsque l'apport ali-

Le texte de cette conférence est issu d'un travail de thèse qui a reçu le Prix de thèse AFECG 2005. (voir www.afecg.org.)

\begin{abstract}
The purpose of our study was to investigate the effects of high doses of ALA provided by linseed oil (containing 50\% ALA) on its own bioavailability and that of its derivatives as well as on lipid metabolism. We investigated in male hamsters the dose/response effects of ALA over a broad range of supply as linseed oil $(1,10,20$ and $41 \%$ of total fatty acids, $F A$, or $0.4,3.6,6.7$ and $14.6 \%$ of total energy intake). ALA was substituted for oleic acid in order to keep constant linoleic acid (LA) and saturated fatty acids which could interfere with the metabolism of n-3 PUFA and lipids, respectively. The capacity of ALA absorption, transport, storage and conversion into EPA had no limitation over the chosen range of dietary intake. However, dietary ALA failed to increase DHA content in plasma phospholipids. In parallel to the increase in EPA, arachidonic acid content decreased, resulting in an improved balance of 20 carbons FA. Moreover, in our atherogenic conditions, triglyceridemia decreased by $45 \%$ in response to $10 \%$ dietary ALA and was not affected by higher intakes. It was associated with lower hepatic activities of acetyl-CoA-carboxylase (up to - 29\%) and malic enzyme (up to - 42\%), which were negatively correlated to ALA intake ( $r^{2}=0.33$ and $r^{2}=0.38$, respectively). Substitution of $10 \%$ ALA for oleic acid increased cholesterolemia by $15 \%$ but, as in TG, higher ALA intakes did not amplify the response. The highest ALA intake (40\%) modified dramatically hepatobiliary metabolism of sterols. Thus, replacing $10 \%$ oleic acid by ALA is sufficient to improve its bioavailability and that of EPA, and to exert a beneficial hypotriglyceridemic effect, that may be counteracted by the slight increase in cholesterolemia. Higher intakes did not modify these parameters, but a very high dose resulted in adverse effects on sterol metabolism and does not seem appropriate for humans.
\end{abstract}

Key words: alpha-linolenic acid, oleic acid, fatty acid bioavailability, lipogenesis, hamster

mentaire du précurseur augmente [7]. II se pourrait donc qu'à de fortes teneurs, l'absorption de l'ALA, son stockage ou sa conversion en dérivés à longue chaîne puissent être limités. Dans ce cas, apporter des doses importantes de cet acide gras (AG) dans I'alimentation serait peu intéressant. Par ailleurs, les études évaluant les effets de l'ALA, I'ont substitué à l'acide linoléique (LA) pour atteindre un large ratio LA/ALA. Cependant, celui-ci influence le métabolisme des AGPI, et notamment la conversion de l'ALA en ses dérivés. Ainsi, les résultats obtenus sont dus à un double effet, celui de l'augmentation des teneurs en ALA et de la diminution de celles en LA.

Outre son intérêt pour augmenter la biodisponibilité des AGPI n-3, I'ALA exerce un potentiel effet bénéfique sur les facteurs lipidiques des MCV. La plupart des études portant sur les effets métaboliques de I'ALA chez I'homme comme chez l'animal divergent. Certaines études montrent que I'ALA alimentaire (apporté à hauteur de 1,8\% de l'apport énergétique total, $A E T$ ) augmente légèrement la triglycéridémie [8] certaines ne montrent aucun effet (avec une consommation de 4-5\% de l'apport énergétique total, AET) $[9,10]$, alors que d'autres révèlent une baisse de la triglycéridé- mie $[11,12]$. Les différences observées entre ces études pourraient provenir de l'hétérogénéité des doses utilisées et du contexte lipidique dans lequel I'ALA se trouve ; en particulier, les rapports LA/ALA et AGS/AGPI sont importants à maîtriser. Il convient alors de s'intéresser aux effets métaboliques de l'ALA apporté à des doses différentes et de s'assurer que cet acide gras n'exerce pas d'effets délétères à forte dose.

Les objectifs de cette étude sont donc multiples, il s'agit dans un premier temps de regarder s'il existe une limite biologique aux phénomènes d'absorption, de transport, de stockage, et de conversion de I'ALA en ses dérivés à longue chaîne, puis, dans un second temps, d'étudier ses effets sur quelques éléments clés du métabolisme des TG et du cholestérol, en accordant une attention particulière aux éventuels effets délétères de cet acide gras lorsqu'il est apporté à forte dose. Pour ce faire, nous avons étudié, chez le hamster, modèle reconnu pour l'étude des acides gras alimentaires sur le métabolisme lipidique [13-15], I'effet doseréponse de I'ALA apporté par l'huile de lin, sur une large gamme d'apport de cet acide gras, tout en maintenant les apports en LA et en AGS constants. La biodisponibilité des AGPIn-3 a été évaluée sur les esters de cholestérol $(E C)$ plas- 
matiques pour le transport de l'ALA, les TG du tissu adipeux épididymaire pour son stockage et les PL plasmatiques pour le transport des AGPI n-3 à longue chaîne. Nous avons de plus analysé la composition lipidique du plasma et du foie, ainsi que quelques enzymes de la lipogenèse hépatique et l'excrétion fécale des stérols.

\section{Matériels et méthodes}

\section{Animaux}

24 hamsters syriens dorés mâles (Mesocricetus auratus) provenant de l'élevage Janvier (Le Genest-St-Isle, France) et âgés de 4 semaines, ont été placés en cages collectives ( 6 animaux par cage) sur litière, dans un environnement contrôlé $\left(22^{\circ} \mathrm{C}\right.$, cycle lumineux $14 \mathrm{~h}$ de jour $/ 10 \mathrm{~h}$ de nuit). Jusqu'à 8 semaines, ils ont reçu ad libitum de l'eau distillée, ainsi que des croquettes du commerce broyées de type UAR 113 (UAR, Villemoisson, France), apportant $59,6 \%$ de glucides, $19,0 \%$ de protéines, $6,0 \%$ de lipides et $5,45 \%$ de minéraux.

\section{Régimes}

Les 4 régimes expérimentaux étaient constitués de 84,4\% (en poids) des croquettes décrites ci-dessus, de $3 \%$ d'eau, de $0,03 \%$ de cholestérol (5-cholesten-3 $\beta$-ol, Sigma, St Louis, États-Unis) et de 12,5\% d'un mélange d'huiles végétales. Les 4 régimes étaient composés de $16,3 \%$ de protéines, $52,6 \%$ de glucides, $16,6 \%$ de lipides, $11,8 \%$ d'eau et $4,6 \%$ de minéraux. Les lipides apportaient environ $1 / 3$ (35,3\%) de l'AET. Les mélanges d'huiles végétales étaient constitués d'huile de lin $53,6 \%$ d'ALA, $15,7 \%$ de LA, $20 \%$ d'acide oléique et

\footnotetext{
Abréviations :

$A A$, acide arachidonique :

$A$, acide biliaire

ACC, acétyl-CoA carboxylase ;

$A E T$, apport énergétique total ;

$A G$, acide gras ;

$A G M I$, acide gras mono-insaturé ;

$A G P I$, acide gras polyinsaturé ;

AGS, acide gras saturé ;

$A L A$, acide $\alpha$-linolénique :

$C L$, cholestérol libre :

$C T$, cholestérol total

$D H A$, acide docosahexaenoïque ;

$D P A$, acide docosapentaenoïque ;

$E C$, esters de cholestérol ;

$E M$, enzyme malique ;

$E P A$, acide eicosapentaenoïque ;

G6PDH, glucose 6 phosphate déshydrogénase ;

$G R$, globule rouge ;

$L A$, acide linoléique ;

PL, phospholipide ;

$T A E$, tissu adipeux épididymaire
}

$10 \%$ d'acides gras saturés, AGS) (Valorex, Javené, France) et d'huile de tournesol oléique (74,9 \% d'acide oléique, 0,1 \% d'ALA, 14,6\% de $L A$, et $10 \%$ d'ACS) ajoutées en proportions variables pour augmenter la concentration d'ALA tout en gardant constantes les concentrations en LA et AGS. Par conséquent, la teneur en ALA diminuait lorsque celle en acide oléique (18:1 n-9) augmentait. Les proportions théoriques d'huile de lin et de tournesol oléique étaient: 0/100, 22/78, 47/53 et 97/3, apportant théoriquement 1, 10, 20, $41 \%$ d'ALA (en $\%$ des AC totaux) soit $0,4,3,6,6,7$ et $14,6 \%$ de l'AET, dans les régimes L1, L10, L20 et $L 40$, respectivement. Ainsi le rapport LA/ALA variait dans une large gamme de 0,5 avec le régime $L 40$ à 22,5 dans le régime L1. La composition des régimes expérimentaux a été déterminée comme décrite ci-dessous et rapportée dans le tableau 1. Tous les régimes apportaient des quantités similaires de lipides, cholestérol et phytostérols de manière à ce que la seule variable soit la composition en acide gras.

\section{Procédure expérimentale}

Après 5 semaines de régime expérimental en cages collectives, les hamsters ont été placés en cages individuelles sur grilles pour mesurer leur consommation et pour collecter leurs fèces durant la $7^{\mathrm{e}}$ semaine. Afin de mesurer le gain de poids et la consommation alimentaire durant la dernière semaine, les hamsters ont été mis à jeun pendant une nuit (18 heures) à la fin de la $6^{\mathrm{e}}$ semaine. À l'âge de 15 semaines, après 7 semaines de régime expérimental, tous les hamsters à jeun d'une nuit, ont été pesés puis anesthésiés par injection intramusculaire de Zoletil 50 (Virbac, Carros, France) (4 mg/100 g de poids corporel). Le sang a été prélevé par ponction intracardiaque grâce à des seringues héparinées (10 unités héparine/mL sang). Le plasma a été séparé des globules rouges (GR) par centrifugation à $1700 \mathrm{~g}$ pendant 20 min à $4{ }^{\circ} \mathrm{C}$ puis stocké à $-20^{\circ} \mathrm{C}$. Les GR ont été rincés au sérum physiologique puis congelés à $-80^{\circ} \mathrm{C}$. Après la prise de sang, la paroi abdominale a été sectionnée et le foie prélevé précautionneusement puis pesé. Un échantillon de $200 \mathrm{mg}$ a été gardé sur la glace pour la préparation du surnageant post-mitochondrial utilisé pour la mesure des activités $\Delta 6$ - et $\Delta 5$ - désaturases. Un autre échantillon de $1 \mathrm{~g}$ de foie a immédiatement été congelé dans l'azote liquide pour les mesures des activités des enzymes de la lipogenèse, et un dernier de $0,5 \mathrm{~g}$ a été stocké à $-20^{\circ} \mathrm{C}$ pour l'analyse de la composition lipidique. Le cœur et le tissu adipeux épididymaire ont été prélevés, pesés et stockés à $-80^{\circ} \mathrm{C}$.

\section{Analyses}

Profiles d'acides gras. Les protocoles de séparation des acides gras ont été décrits par Morise et al. [16].

Mesure de l'excrétion fécale des stérols. Les stérols neutres (dont le cholestérol) et acides (aides biliaires) ont été extraits des fèces d'après une méthode décrite par Boehler et al. [17]. Mesure des activités hépatiques des $\Delta 6$ - et 45-désaturases. Cette mesure a été établie d'après la technique décrite par Guillou et al. [18].

Composition du plasma et du foie. L'analyse des lipides sanguins et hépatiques a été décrite par Morise et al. [19].

Mesure des activités des enzymes de la lipogenèse hépatiques. Les activités des enzymes de la lipogenèse ont été évaluées selon une méthode décrite par Mourot et al. [20].

Tableau 1. Composition lipidique des régimes expérimentaux.

\begin{tabular}{|c|c|c|c|c|c|}
\hline & Unité & $\mathbf{L 1}$ & L10 & L20 & L40 \\
\hline Lipides totaux & $\mathrm{g} / 100 \mathrm{~g}$ & 15,7 & 16,0 & 14,9 & 15,6 \\
\hline Cholestérol & Régime & 0,072 & 0,075 & 0,075 & 0,072 \\
\hline$\Sigma$ AGS & $\%$ acides gras & 10,96 & 10,4 & 11,54 & 11,65 \\
\hline$\Sigma$ AGMI & totaux & 64,07 & 55,83 & 44,29 & 22,18 \\
\hline dont $18: 1 n-9$ & & 63,74 & 55,47 & 43,95 & 21,79 \\
\hline$\Sigma$ AGPI & & 24,95 & 33,77 & 44,19 & 66,22 \\
\hline$\Sigma \mathrm{n}-6$ & & 23,65 & 23,48 & 24,28 & 24,55 \\
\hline dont $18: 2 n-6$ & & 23,65 & 23,48 & 24,28 & 24,55 \\
\hline$\Sigma \mathrm{n}-3$ & & 1,30 & 10,29 & 19,91 & 41,67 \\
\hline dont $18: 3 n-3$ & & 1,05 & 9,95 & 19,50 & 41,30 \\
\hline $18: 2 n-6 / 18: 3 n-3$ & & 22,52 & 2,36 & 1,25 & 0,59 \\
\hline $18: 3 n-3$ & $\%$ AET & 0,4 & 3,6 & 6,7 & 14,6 \\
\hline
\end{tabular}

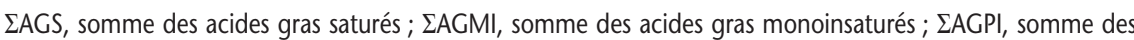
acides gras polyinsaturés; AGPI/AGS, ratio des AGPI to AGS; $\Sigma \mathrm{n}-6$, somme des AGPI n- 6 ; $\Sigma \mathrm{n}-3$, somme des AGPI n-3. 


\section{Analyses statistiques}

Les données ont été analysées au moyen du logiciel Statview 4.5 (Abacus Concept, Berkeley, CA, États-Unis), en faisant appel à une Anova suivie d'un test de Fisher a posteriori. Les différences entre moyennes ont été considérées comme significatives au seuil de 0,05.

\section{Résultats}

\section{Croissance et absorption apparente des lipides}

Le poids des hamsters à la fin des 7 semaines de régime expérimental diffère légèrement mais significativement entre les animaux du groupe $\mathrm{L} 1$, les plus lourds ( $120 \mathrm{~g}$ ), et ceux des groupes $\mathrm{L} 20$ et $\mathrm{L} 40$, les plus légers $(110 \mathrm{~g})$. Cette différence de poids n'est cependant pas associée à une différence de consommation alimentaire ou de croissance corporelle lors de la dernière semaine d'expérimentation. Elle ne se ressent pas non plus sur le poids du tissu adipeux épididymaire qui est identique dans les 4 groupes [19].

L'absorption apparente des lipides est très élevée (environ $95 \%$ ) dans tous les groupes, cependant, les animaux des groupes L1 et L20 ont une absorption plus élevée de $1 \%$ que ceux du groupe L40. L'absorption des AC est elle aussi très élevée. Celle de l'acide oléique et de I'ALA est plus faible chez les animaux du groupe L40 et L1 respectivement. Au contraire, I'absorption du LA et des AGS ne diffère pas entre les groupes [19].

\section{Enrichissement en ALA des différents tissus}

Dans tous les tissus étudiés (figure 1), I'enrichissement en ALA est proportionnel à l'ingestion de cet acide gras. Le tissu adipeux épididymaire et les EC plasmatiques s'enrichissent beaucoup plus en ALA que les PL plasmatiques ou cardiaques, ou les GR. Les coefficients de régression linéaire sont tous très élevés entre 0,97 pour les $\mathrm{PL}$ et $\mathrm{EC}$ plasmatiques et 0,99 pour les $\mathrm{GR}$, la probabilité étant inférieure à 0,0001 dans tous les cas.

\section{Profil d'acide gras des PL plasmatiques}

Parallèlement à l'enrichissement des PL en ALA, on observe un enrichissement en EPA et DPA mais pas en DHA (figure 2). La proportion de ce dernier diminue même lorsque la quantité d'ALA absorbée est très élevée (groupes L20 et L40). Tout comme l'enrichissement en ALA, celui en EPA est proportionnel à l'ingestion $d^{\prime} A L A\left(R^{2}=0,93, P<0,0001\right)$, alors que celui en DPA plafonne à partir de $10 \%$ d'ALA dans le régime. Par ailleurs, bien que la teneur en LA soit identique dans tous les régimes, la propor-
ALA

(\% des acides gras totaux)

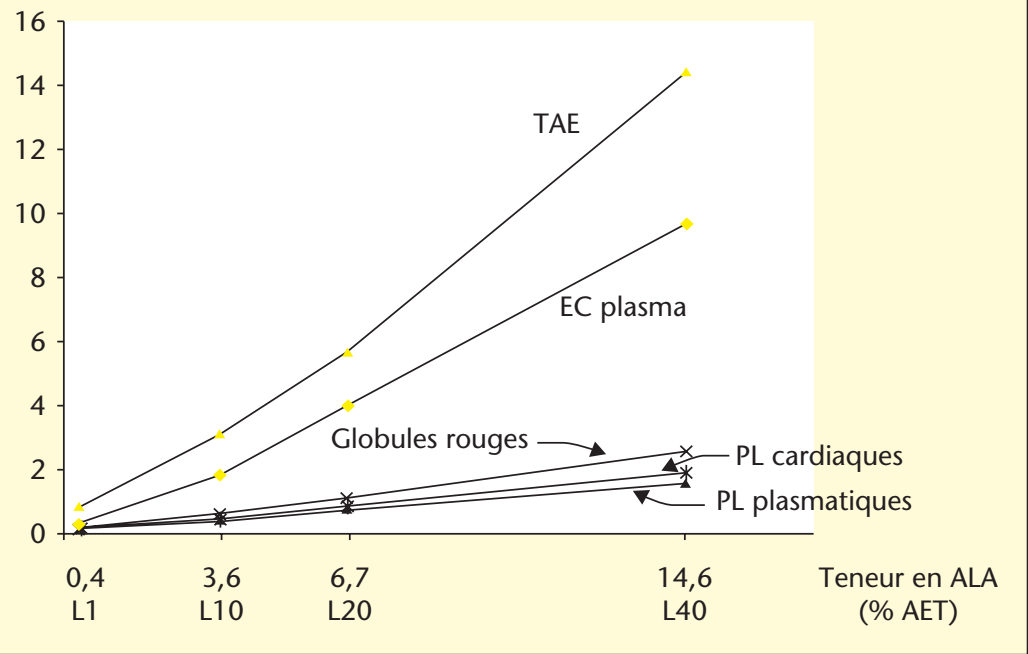

Figure 1. Proportion d'ALA dans les différents tissus en fonction de l'apport alimentaire d'ALA. EC: esters de cholestérol, PL : phospholipides, TAE : tissu adipeux épididymaire, AET : apport énergétique total.

tion de LA dans les PL plasmatiques augmente lorsque l'apport en ALA augmente $\left(22,28 \pm 0,37^{c}, 24,41 \pm 0,16^{c}, 24,48 \pm 0,64^{b}\right.$, $27,47 \pm 0,25^{\text {a }}$, respectivement dans les groupes $\mathrm{L} 1, \mathrm{~L} 10, \mathrm{~L} 20$ et $\mathrm{L} 40$ ) tandis que celle en $\mathrm{AA}$ diminue $\quad\left(8,74 \pm 0,32^{\mathrm{a}}, \quad 7,10 \pm 0,11^{\mathrm{b}}\right.$, $7,12 \pm 0,34^{\mathrm{b}}, 6,28 \pm 0,13^{\mathrm{c}}$, respectivement dans les groupes L1, L10, L20 et L40) (figure 3).

\section{Activités des 45 - et 46 -désaturases hépatiques}

Augmenter les apports alimentaires d'ALA n'a pas eu d'effet sur les activités des $\Delta 5$ - et $\Delta 6$-désaturases hépatiques qui étaient identiques dans tous les groupes qui s'élevaient à 377 et à $208 \mathrm{pmol}$ de substrat $/ \mathrm{min} / \mathrm{mg}$ de

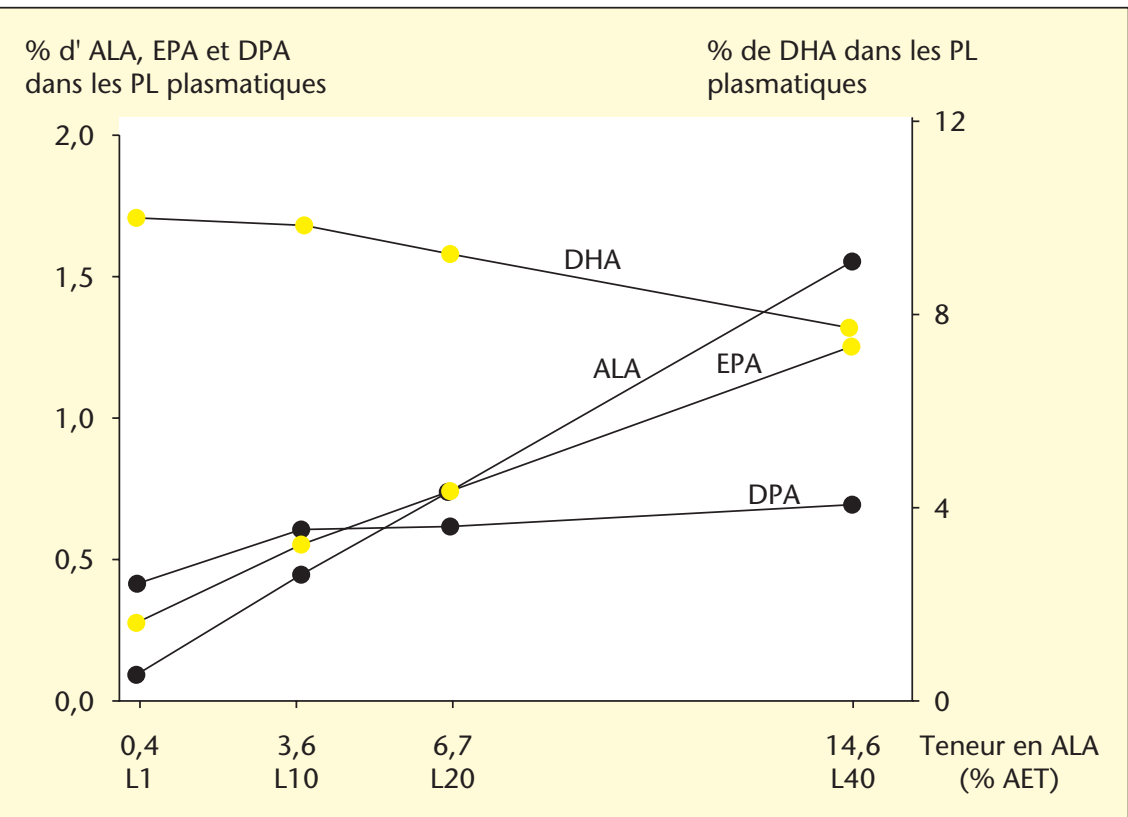

Figure 2. Proportion d'AGPI n-3 dans les PL plasmatiques en fonction de l'apport alimentaire d'ALA. ALA : acide $\alpha$-linolénique, EPA : acide eicosapentaenoïque, DHA : acide docosahexaenoïque, PL : phospholipides, AET : apport énergétique total. 


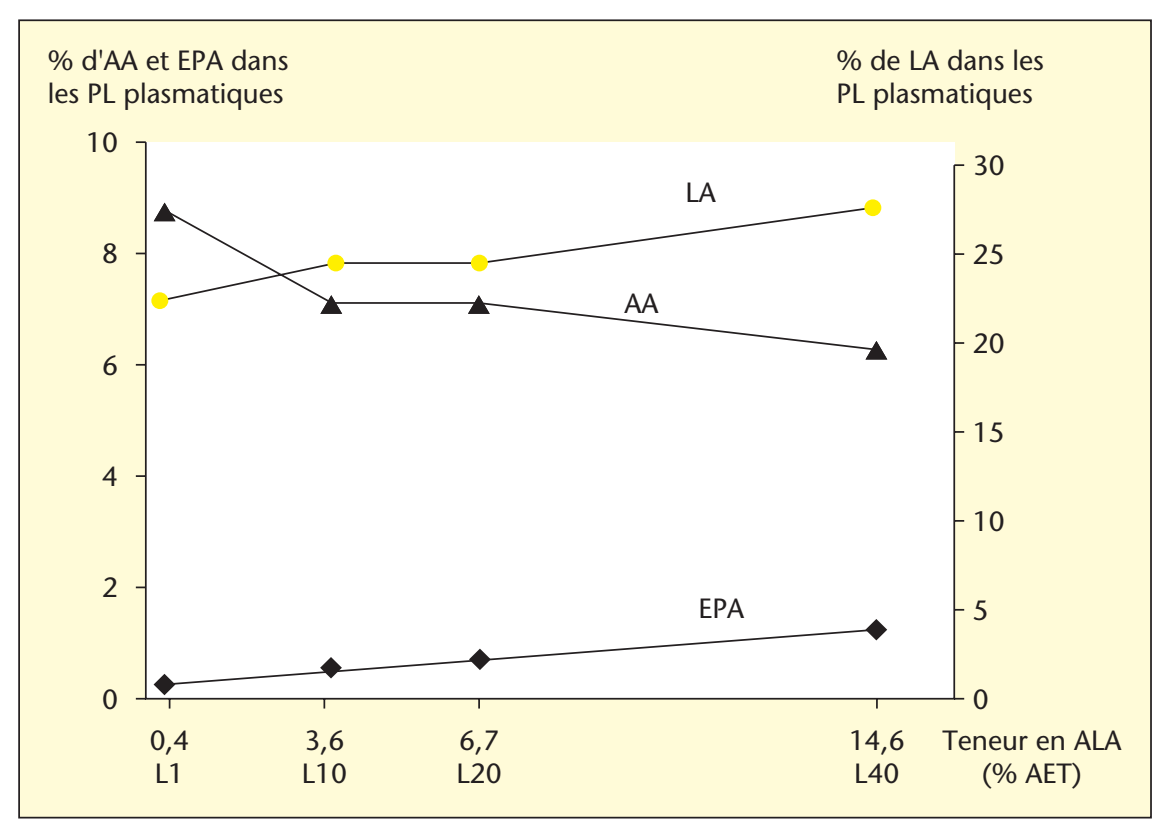

Figure 3. Proportion d'AGPI n-6 dans les PL plasmatiques en fonction de l'apport alimentaire d'ALA. AA : acide arachidonique, AET : apport énergétique total, LA : acide linoléique, EPA : acide eicosapentaenoïque, PL : phospholipides.

Tableau 2. Lipides plasmatiques ( $\mathrm{g} / \mathrm{L})$.

\begin{tabular}{|lcccc|}
\hline & $\mathbf{L 1}$ & $\mathbf{L 1 0}$ & $\mathbf{L 2 0}$ & $\mathbf{L 4 0}$ \\
\hline TC & $1,62 \pm 0,02^{\mathrm{b}}$ & $1,86 \pm 0,06^{\mathrm{a}}$ & $1,91 \pm 0,06^{\mathrm{a}}$ & $1,81 \pm 0,09^{\mathrm{a}}$ \\
TG & $1,52 \pm 0,26^{\mathrm{a}}$ & $0,83 \pm 0,05^{\mathrm{b}}$ & $0,72 \pm 0,14^{\mathrm{b}}$ & $0,94 \pm 0,15^{\mathrm{b}}$ \\
PL & $2,80 \pm 0,05^{\mathrm{a}}$ & $2,51 \pm 0,07^{\mathrm{b}}$ & $2,32 \pm 0,05^{\mathrm{c}}$ & $2,21 \pm 0,07^{\mathrm{c}}$ \\
HDL-C & $1,18 \pm 0,13$ & $1,30 \pm 0,07$ & $1,23 \pm 0,12$ & $1,16 \pm 0,11$ \\
HDL-PL & $1,81 \pm 0,05$ & $1,97 \pm 0,06$ & $1,73 \pm 0,11$ & $1,62 \pm 0,06$ \\
\hline Non HDL-C/HDL-C & $0,39 \pm 0,06$ & $0,44 \pm 0,03$ & $0,61 \pm 0,12$ & $0,60 \pm 0,12$ \\
\hline
\end{tabular}

Moyenne \pm erreur standard, $n=6$. Des lettres différentes indiquent une différence significative entre groupes au seuil de 5 \%. CT : cholestérol total ; PL : phospholipides ; TG : triglycérides.

Tableau 3. Composition du foie.

\begin{tabular}{|c|c|c|c|c|c|}
\hline & Unité & $\mathbf{L 1}$ & L10 & $\mathbf{L 2 0}$ & L40 \\
\hline \multirow{2}{*}{$\begin{array}{l}\text { Poids } \\
\text { du foie }\end{array}$} & g & $4,10 \pm 0,09$ & $3,94 \pm 0,05$ & $3,84 \pm 0,08$ & $3,90 \pm 0,14$ \\
\hline & $\%$ de masse corporelle & $3,53 \pm 0,06$ & $3,49 \pm 0,04$ & $3,44 \pm 0,07$ & $3,40 \pm 0,04$ \\
\hline $\mathrm{CT}$ & $\mathrm{mg} / \mathrm{g}$ de foie & $40,1 \pm 1,9^{a}$ & $41,0 \pm 4,7^{a}$ & $39,9 \pm 4,1^{a}$ & $22,3 \pm 1,7^{b}$ \\
\hline $\mathrm{CL}$ & & $3,27 \pm 0,19^{a}$ & $3,81 \pm 0,39^{a}$ & $3,45 \pm 0,25^{a}$ & $2,41 \pm 0,08^{b}$ \\
\hline EC & & $61,6 \pm 2,8^{a}$ & $62,1 \pm 7,3^{\mathrm{a}}$ & $60,8 \pm 6,5^{a}$ & $33,3 \pm 2,7^{b}$ \\
\hline TG & & $8,34 \pm 0,64^{\mathrm{ab}}$ & $9,50 \pm 0,50^{a}$ & $7,67 \pm 0,51^{b}$ & $8,56 \pm 0,47^{a b}$ \\
\hline PL & & $18,2 \pm 0,4^{a}$ & $18,0 \pm 0,6^{\mathrm{ab}}$ & $15,6 \pm 2,1^{b}$ & $9,0 \pm 0,3^{c}$ \\
\hline PR & & $184 \pm 11^{a}$ & $190 \pm 4^{a}$ & $183 \pm 4^{a}$ & $194 \pm 3^{a}$ \\
\hline
\end{tabular}

Moyenne \pm erreur standard, $\mathrm{n}=6$. Des lettres différentes indiquent une différence significative entre groupes au seuil de $5 \%$. CT : cholestérol total ; CL : cholestérol libre ; EC : esters de cholestérol ; PL : phospholipides ; PR : protéines; TG : triglycérides.

protéines pour les $\Delta 6$ - et $\Delta 5$-désaturase respectivement [21].

\section{Composition lipidique du plasma et du foie (tableaux 2 et 3)}

La concentration en CT plasmatique est plus élevée de $15 \%$ dans tous les groupes ayant nelle à la dose d'ALA ingéré. La concentration en HDL-C et l'index d'athérogénicité (non HDL-C/HDL-C) sont identiques dans les 4 groupes malgré une légère augmentation non significative en réponse aux forts apports d'ALA (groupes L20 et L40).

Dans le foie, les teneurs en $C T, C L$ et $E C$ sont presque deux fois plus faibles chez les animaux nourris au régime L40 (tableau 3). La teneur en PL est elle aussi plus faible chez ces animaux. Enfin, la teneur en TG varie entre les groupes, mais de manière indépendante de la dose d'ALA alimentaire.

\section{Activités des enzymes de la lipogenèse hépatiques}

L'activité spécifique de l'acétyl-coenzymeA carboxilase (ACC) diminue lorsque l'apport alimentaire en ALA augmente, puis plafonne pour une consommation d'ALA de 6,7 \% de l'apport énergétique total (avec le régime L20) (figure 4). De la même façon, l'activité de I'enzyme malique (EM), un fournisseur de NADPH pour la synthèse des acides gras, est inversement proportionnelle à la consommation d'ALA. Cependant, contrairement à celle de I'ACC, I'activité de I'EM ne tend pas à plafonner avec les forts apports en ALA. Au contraire, la glucose6 phosphate déshydrogénase (G6PDH), un autre fournisseur de NADPH, n'est pas influencée par la teneur en ALA des régimes: son activité est identique dans tous les groupes et 4 fois inférieure à celle de l'EM. L'effet de la dose d'ALA ne diffère pas fondamentalement si I'on considère les activités totales par foie [19]. De plus, on peut établir une corrélation significative entre la triglycéridémie et l'activité totale de I'ACC et de I'EM dans le foie $\left(R^{2}=0,17, P=0,04\right.$ et $R^{2}=0,38$, $P=0,002$, respectivement).

\section{Excrétion fécale du cholestérol et des acides biliaires}

Les animaux ayant reçu le régime $\mathrm{L} 40$ ont une excrétion fécale de CT nettement plus élevée (+ $40 \%)$ et d'acides biliaires nettement plus faible $(-60 \%)$ que les animaux des 3 autres groupes (tableau 4). Outre la quantité, la qualité des acides biliaires est elle aussi profondément modifiée par le régime L40. En effet, en réponse à ce régime, les acides biliaires primaires augmentent aux dépens des aides biliaires secondaires, tandis que les acides issus de la voie de l'acide chénodésoxycholique sont favorisés aux dépens de ceux issus de la voie de l'acide cholique. 


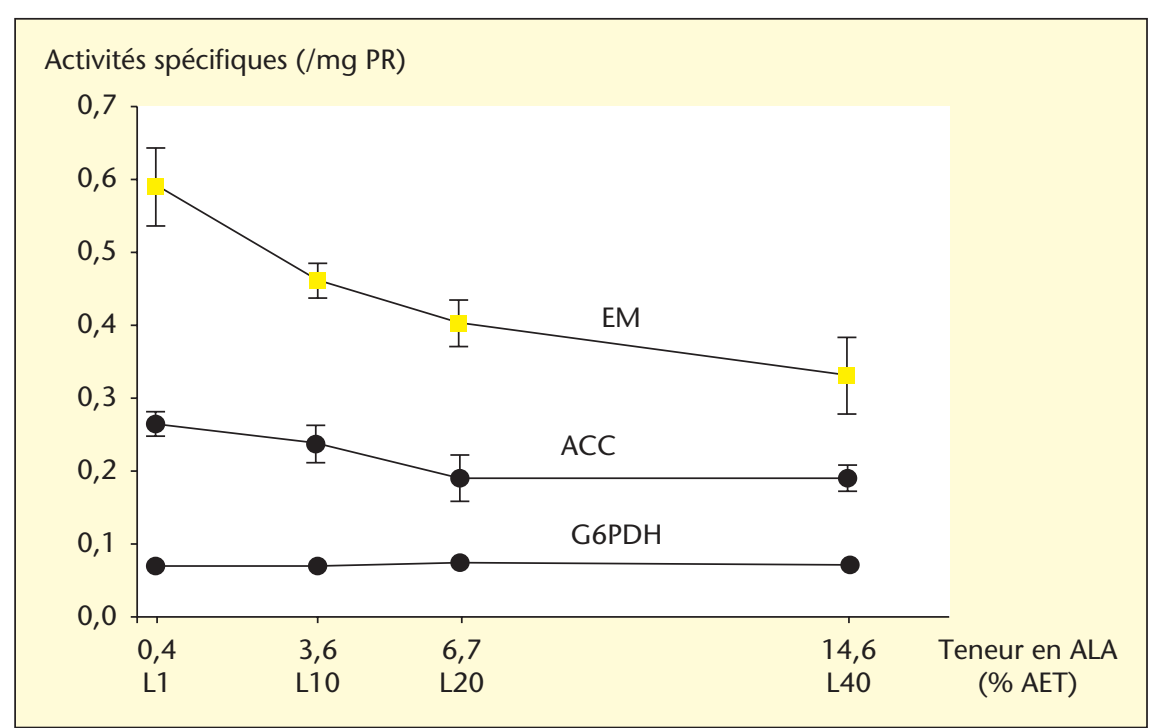

Figure 4. Activités spécifiques des enzymes de la lipogenèse hépatique en fonction de l'apport alimentaire d'ALA. L'activité spécifique de l'EM et de la G6PDH est exprimée en $\mu$ mol de NADPH produite par minute et par mg de protéines. Celle de l'ACC est exprimée en nmol de HCO3-par minute et par mg de protéines. Les résultats sont la moyenne \pm erreur standard, $n=6$. ACC: acétyl-CoA carboxylase, AET: apport énergétique total, EM: enzyme malique, G6PDH : glucose 6 phosphate déshydrogénase.

\section{Discussion}

\section{Biodisponibilité de l'ALA et de ses dérivés}

Dans notre étude, une très large gamme $d^{\prime}$ apport d'ALA est utilisée, allant bien au-delà des doses recommandées (0,8 \% AET). Cependant, aucun phénomène de saturation n'est observé, au niveau de l'absorption de l'ALA, de son transport par les EC plasmatiques ou de son stockage dans le TAE, même pour les doses les plus importantes. En effet ces tissus s'enrichissent en ALA proportionnellement à son apport alimentaire et sans plafonnement pour les fortes doses (figure 1). Ceci est particulièrement intéressant concernant le tissu adipeux puisqu'il représente un des pools d'ALA les plus importants et peut être mobilisable par l'organisme lorsqu'il en a besoin [22]. Ceci suggère que l'organisme peut tirer profit d'apports importants et discontinus d'ALA en le stockant et en le remobilisant.

Parallèlement à un enrichissement en ALA, les PL plasmatiques s'enrichissent en EPA lorsque la consommation d'ALA augmente, alors que la proportion de DPA semble plafonner (figure 2). Ceci reflète l'équilibre complexe entre le stockage de I'EPA, son élongation en DPA, la conversion de celui-ci en $\mathrm{DHA}$, et la rétroconversion du DHA en DPA puis EPA [23]. La réponse du DHA aux apports croissants d'ALA est très particulière. Contrairement à I'EPA et au DPA, la proportion de DHA dans les tissus n'augmente pas avec l'ALA, mais au contraire, diminue avec les plus fortes doses (20-40\% $\left.d^{\prime} A L A\right)$. Ceci s'observe aussi dans les PL cardiaques et dans les globules rouges [21], et semble être un phénomène général dans les tissus

Tableau 4. Excrétion fécale des stérols.

\begin{tabular}{|lccccc|}
\hline Stérol & Unité & $\mathbf{L 1}$ & $\mathbf{L 1 0}$ & $\mathbf{L 2 0}$ & $\mathbf{L 4 0}$ \\
\hline $\mathrm{CT}$ & $\mathrm{mg} / \mathrm{j}$ & $1,25 \pm 0,04^{\mathrm{b}}$ & $1,26 \pm 0,07^{\mathrm{b}}$ & $1,12 \pm 0,07^{\mathrm{b}}$ & $1,53 \pm 0,06^{\mathrm{a}}$ \\
$\mathrm{AB}$ excrété & & $1,51 \pm 0,14^{\mathrm{a}}$ & $1,21 \pm 0,16^{\mathrm{a}}$ & $1,20 \pm 0,11^{\mathrm{a}}$ & $0,50 \pm 0,04^{\mathrm{b}}$ \\
\hline $\mathrm{CT} \pm \mathrm{AB}$ excrétés & $\mu \mathrm{mol} / \mathrm{j}$ & $6,32 \pm 0,37^{\mathrm{a}}$ & $5,72 \pm 0,40^{\mathrm{ab}}$ & $5,35 \pm 0,40^{\mathrm{ab}}$ & $4,97 \pm 0,15^{\mathrm{b}}$ \\
\hline $\mathrm{ABI}$ & $\%$ & $3,59 \pm 0,46^{\mathrm{b}}$ & $4,62 \pm 0,45^{\mathrm{b}}$ & $3,62 \pm 0,71^{\mathrm{b}}$ & $10,66 \pm 1,19^{\mathrm{a}}$ \\
$\mathrm{ABII} / \mathrm{ABI}$ & & $30,0 \pm 4,3^{\mathrm{a}}$ & $22,0 \pm 2,7^{\mathrm{a}}$ & $31,3 \pm 5,1^{\mathrm{a}}$ & $8,9 \pm 1,0^{\mathrm{b}}$ \\
\hline $\begin{array}{l}\text { Voie cholique } \\
\text { Voie cholique/voie }\end{array}$ & $\%$ & $56,4 \pm 0,6^{\mathrm{a}}$ & $54,1 \pm 2,3^{\mathrm{a}}$ & $55,6 \pm 1,4^{\mathrm{a}}$ & $44,9 \pm 1,8^{\mathrm{b}}$ \\
chénodésoxycholique & & $1,30 \pm 0,04^{\mathrm{a}}$ & $1,21 \pm 0,10^{\mathrm{a}}$ & $1,26 \pm 0,07^{\mathrm{a}}$ & $0,83 \pm 0,06^{\mathrm{b}}$ \\
\hline
\end{tabular}

Moyenne \pm erreur standard, $n=6$. Des lettres différentes indiquent une différence significative entre groupes au seuil de $5 \%$. ABI : acides biliaires primaires; $A B I I$ : acides biliaires secondaires; voie cholique : voie de l'acide cholique ; voie chénodésoxycholique : voie de l'acide chénodésoxycholique.

périphériques. L'effet observé de I'ALA alimentaire sur la proportion de DHA est en accord avec les études chez l'homme, où des doses modérées d'ALA sont sans effets sur la proportion de DHA dans les tissus [24]. Ils sont aussi cohérents avec les études chez l'animal où des doses importantes d'ALA ont diminué la proportion de DHA [25].

L'enrichissement des tissus en ALA et EPA est proportionnel à l'apport d'ALA, ce qui suggère que la répartition de I'ALA entre $\beta$-oxydation et conversion en ses dérivés n'est pas modifiée par son apport, même lorsqu'il est élevé. Cette hypothèse est appuyée par le fait que l'activité des désaturases responsables de la conversion de l'ALA en ses dérivés n'est pas affectée par la dose d'ALA dans les régimes; ces enzymes ne sont donc ni activées par leur substrat, ni inhibées par leur produit.

Si les doses importantes d'ALA ne limitent pas sa propre conversion en EPA, elles limitent vraisemblablement celle du LA en AA. En effet, alors que les régimes contiennent tous la même quantité de $L A$, la proportion de $L A$ dans les $\mathrm{PL}$ plasmatiques augmente, et celle en AA diminue avec la consommation d'ALA (figure 3). Ceci suggère que lorsque les apports d'ALA augmentent, la compétition entre LA et ALA pour la $\Delta 6$-désaturase devient plus importante, et comme l'ALA est son substrat préférentiel [26], le LA est moins bien converti en AA. Ainsi, la consommation d'ALA augmente le rapport $E P A / A A$, et par conséquent, la proportion des eicosanoïdes de la série 3 aux propriétés antiinflammatoires et limitant l'agrégation plaquettaire (donc anti-athérogènes) par rapport à ceux de la série 6 plus athérogènes.

\section{Effets des doses croissantes d'ALA sur le métabolisme lipidique}

Dans notre étude où les animaux étaient nourris avec des régimes athérogènes riches en lipides et en cholestérol, I'ALA exerce un net effet hypotriglycéridémiant (- $45 \%$ ) qui apparaît dès que l'ALA atteint $3,6 \%$ de l'AET ou (10\% des acides gras totaux). Cependant, chez l'homme, l'effet hypotriglycéridémiant observé avec des doses équivalentes (de 1 à $6 \%$ de l'AET), est moins important $[11,12]$. Cet effet $n^{\prime}$ est pas amplifié par les doses plus fortes d'ALA. Ces résultats sont validés par les modifications des activités de deux enzymes de la lipogenèse hépatique, I'ACC (enzyme clé de la synthèse des $A G$ ) et l'EM (fournisseur de $\mathrm{NADPH}$, indispensable à la synthèse des $A G$ ), qui sont diminuées de manière inversement proportionnelle aux apports d'ALA. Ceci rejoint certaines études chez le Rat où l'administration d'ALA diminue l'activité de lipogenèse [27, 28]. Ainsi, une corrélation entre la triglycéridémie et l'activité des enzymes de la lipogenèse a pu être établie. 
Dans la plupart des études, la substitution de I'ALA aux AGS, entraîne une baisse du CT plasmatique [12]. Dans notre étude cependant, I'ALA est substitué à un acide gras monoinsaturé l'acide oléique (C18:1), et augmente la cholestérolémie de $15 \%$, et ce, dès les doses les plus faibles d'ALA. Cependant, un apport plus important d'ALA n'amplifie pas cet effet. L'augmentation de $\mathrm{CT}$ touche à la fois le HDL-C et le non HDL-C, mais l'effet est plus prononcé sur le non HDL-C; ainsi, le ratio non HDL$\mathrm{C} / \mathrm{HDL}-\mathrm{C}$ tend à augmenter pour les valeurs les plus fortes d'ALA, mais ce n'est pas significatif. Il faut toutefois préciser que l'acide oléique est connu pour augmenter la concentration plasmatique en HDL-C $[29,30]$. II se pourrait donc que la tendance à l'augmentation du ratio non HDL-C/HDL-C suite à l'ingestion des fortes doses d'ALA soit due à la baisse de la teneur en acide oléique des régimes. Dans les études chez I'homme, I'ALA, substitué aux AGS, diminue de manière équivalente le LDL-C et le HDL-C n'affectant pas l'indice d'athérogénicité [12]. La légère augmentation de la cholestérolémie observée chez les animaux recevant de I'ALA, n'est due ni à un plus faible stockage de CT dans le foie ni à une plus forte excrétion fécale de $\mathrm{CT}$ qui sont identique dans ces 3 groupes (tableaux 3 et 4). Cette étude ne permet malheureusement pas d'expliciter pourquoi l'ALA alimentaire induit une cholestérolémie plus élevée que l'acide oléique. Différentes étapes clés du métabolisme des stérols peuvent être impliquées comme la synthèse du cholestérol et des acides biliaires ou la captation du cholestérol par le foie via les récepteurs aux lipoprotéines. Contrairement aux autres paramètres sensibles aux faibles doses d'ALA, la teneur en CT hépatique n'est pas modifiée par les apports d'ALA les plus faibles, mais diminue de $50 \%$ en réponse au régime qui en est le plus riche $(\mathrm{L} 40)$. Ce même régime induit de plus, une augmentation de $30 \%$ de l'excrétion fécale de CT et une baisse de $60 \%$ de celle des acides biliaires. Outre la quantité d'acides biliaires excrétés, leur qualité est elle aussi affectée : les proportions entre la voie de l'acide cholique et celle du chénodésoxycholique sont inversées, augmentant ainsi l'hydrophobicité de la bile et donc la susceptibilité des hamsters à développer des calculs biliaires. Ainsi, dans notre modèle hamster, les apports d'ALA très élevés (et non physiologiques) présentent un danger potentiel pour le métabolisme hépatobiliaire.

\section{Conclusion}

Dans nos conditions expérimentales, I'ALA alimentaire permet d'augmenter la biodisponibilité de cet $A G$ et de certains de ses dérivés. En effet, les phénomènes d'absorption, de transport, de stockage de l'ALA ne sont pas saturés par de forts apports en cet AG. De plus, si la consommation d'ALA n'augmente pas la teneur en DHA des tissus étudiés, elle augmente celle en EPA, tout en diminuant celle en AA. Ceci est particulièrement intéressant, pour la prévention des MCV notamment, puisque I'EPA est le précurseur des eicosanoïdes de la série 3 connus pour leur rôle protecteur de cette pathologie, contrairement à ceux de la série 2 issus de l'AA.

L'ALA pourrait de plus exercer un effet bénéfique sur les facteurs de risque des MCV. En effet, bien que, comparé à l'acide oléique, l'ALA augmente de $15 \%$ la cholestérolémie (sans modifier de manière significative l'indice d'athérogénicité), il diminue, dans nos conditions athérogènes, la triglycéridémie via une baisse de l'activité hépatique des enzymes de la lipogenèse chez le hamster. Ces effets sont observés avec des doses relativement faibles (10\% des acides gras totaux ou 3,6 \% de l'AET) et ne sont pas amplifiés par des doses plus importantes. Ainsi, les résultats contradictoires rapportés dans la littérature sur les différents effets de I'ALA sur la triglycéridémie ne semblent pas être dus à la dose d'ALA, au moins entre 3,6 et $14,6 \%$ de l'AET. En revanche, la diversité des AG auxquels on substitue I'ALA pourrait expliquer cette variabilité.

Si les doses modérées semblent être potentiellement bénéfiques, les très fortes doses non physiologiques testées ici ne sont pas recommandables (ni applicables à l'homme) puisque, dans notre modèle hamster, non seulement elles diminuent la teneur en DHA des PL plasmatiques mais encore, elles perturbent le métabolisme hépatobiliaire.

Remerciements. Nous remercions vivement I'Onidol et I'Oniol (Office national interprofessionnel des oléagineux, protéagineux et cultures textiles) pour leur soutien financier, Guillaume Chesneau et Valorex (La Messayais, 35210 Combourtillé, France) pour avoir fourni I'huile de lin indispensable à cette étude, ainsi que Gwennola Robin (Senah) pour sa contribution aux mesures des activités des enzymes de la lipogenèse. Nous remercions aussi Bernadette Delplanque (Université Paris Sud) pour sa lecture avisée de l'article et pour ses précieux conseils scientifiques.

\section{REFERENCES}

1. NORDOY A, et al. n-3 polyunsaturated fatty acids and cardiovascular diseases. Lipids 2001 ; 36(Suppl) : S127-S129.

2. RENAUD S, et al. Cretan Mediterranean diet for prevention of coronary heart disease. Am / Clin Nutr 1995 ; 61(6 Suppl) : 1360S-1367S.

3. DE LORGERIL M, et al. Mediterranean alphalinolenic acid-rich diet in secondary prevention of coronary heart disease. Lancet 1994 ; 343(8911) : 1454-9.
4. COMBE N, BOUE C. Apports alimentaires en acide linoleique et alpha-linolénique d'une population d'Aquitaine. OCL $2001 ; 8(2)$ : 11821.

5. HAREL Z, et al. Omega-3 polyunsaturated fatty acids in adolescents: knowledge and consumption. J Adolesc Health $2001 ; 28(1)$ : 10-5.

6. KRIS-ETHERTON PM, et al. Polyunsaturated fatty acids in the food chain in the United States. Am / Clin Nutr 2000; 71(1 Suppl): 179S-188S.

7. VERMUNT SH, et al. Effects of dietary alphalinolenic acid on the conversion and oxidation of 13C-alpha-linolenic acid. Lipids 2000 ; 35(2) : 137-42.

8. BEMELMANS W], et al. Effect of an increased intake of alpha-linolenic acid and group nutritional education on cardiovascular risk factors : the Mediterranean Alpha-linolenic Enriched Groningen Dietary Intervention (MARGARIN) study. Am / Clin Nutr 2002 ; 75(2) : 221-7.

9. MANTZIORIS E, et al. Dietary substitution with an alpha-linolenic acid-rich vegetable oil increases eicosapentaenoic acid concentrations in tissues. Am / Clin Nutr 1994 ; 59(6) : 1304-9.

10. NYDAHL M, et al. Similar serum lipoprotein cholesterol concentrations in healthy subjects on diets enriched with rapeseed and with sunflower oil. Eur / Clin Nutr 1994 ; 48(2) : 128-37.

11. SINGER P, WIRTH M, BERGERI. A possible contribution of decrease in free fatty acids to low serum triglyceride levels after diets supplemented with n- 6 and $\mathrm{n}-3$ polyunsaturated fatty acids. Atherosclerosis $1990 ; 83(2-3)$ : 167-75.

12. WILKINSON P, et al. Influence of alphalinolenic acid and fish-oil on markers of cardiovascular risk in subjects with an atherogenic lipoprotein phenotype. Atherosclerosis 2005 ; 181(1) : 115-24.

13. SURETTE ME, et al. Dietary $\mathrm{n}$ - 3 polyunsaturated fatty acids modify Syrian hamster platelet and macrophage phospholipid fatty acyl composition and eicosanoid synthesis : a controlled study. Biochim Biophys Acta 1995 ; 1255(2): 185-91.

14. SURETTE ME, et al. Dependence on dietary cholesterol for n-3 polyunsaturated fatty acidinduced changes in plasma cholesterol in the Syrian hamster. / Lipid Res 1992 ; 33(2) : 26371.

15. SPADY DK, KEARNEY DM, HOBBS HH. Polyunsaturated fatty acids up-regulate hepatic scavenger receptor $\mathrm{B} 1$ (SR-BI) expression and $\mathrm{HDL}$ cholesteryl ester uptake in the hamster. / Lipid Res $1999 ; 40(8)$ : 1384-94.

16. MORISE A, et al. Effects of dietary alpha linolenic acid on cholesterol metabolism in male and female hamsters of the LPN strain. / Nutr Biochem $2004 ; 15(1)$ : 51-61. 
17. BOEHLER N, et al. Antilithiasic effect of betacyclodextrin in LPN hamster : comparison with cholestyramine. / Lipid Res 1999 ; 40(4) : 72634.

18. GUILLOU H, et al. Comparative effect of fenofibrate on hepatic desaturases in wild-type and peroxisome proliferator-activated receptor alpha-deficient mice. Lipids 2002; 37(10): 981-9.

19. MORISE A, et al. Dose effect of alpha-linolenic acid on lipid metabolism in the hamster. Reprod Nutr Dev 2005 ; 45(4) : 405-18.

20. MOUROT J, et al. Role of hepatic lipogenesis in the susceptibility to fatty liver in the goose (Anser anser). Comp Biochem Physiol B Biochem Mol Biol $2000 ; 126(1)$ : 81-7.

21. MORISE A, et al. Dose effect of alpha-linolenic acid on PUFA conversion, bioavailability and storage in the Hamster. Lipids 2004 ; 39(4) : 325-34.
22. SINCLAIRAJ FZ. Increased alpha-linolenic acid intake increases tissue alpha-linolenic acid content and apparent oxidation with little effect on tissue docosahexaenoic acid in the guinea pig. Lipids $2000 ; 35(4)$ : 395-400.

23. SPRECHER $\mathrm{H}$. The roles of anabolic and catabolic reactions in the synthesis and recycling of polyunsaturated fatty acids. Prostaglandins Leukot Essent Fatty Acids 2002 ; 67(2-3) : 79-83.

24. GERSTER H. Can adults adequately convert alpha-linolenic acid (18:3n-3) to eicosapentaenoic acid (20:5n-3) and docosahexaenoic acid (22: 6n-3)? Int / Vitam Nutr Res 1998 ; 68(3) : 159-73.

25. BLANK C, et al. Optimizing DHA levels in piglets by lowering the linoleic acid to alphalinolenic acid ratio. / Lipid Res 2002 ; 43(9) : 1537-43.
26. GEIGER M, et al. Studies to determine if rat liver contains chain-length-specific acyl-CoA 6-desaturases. Biochim Biophys Acta 1993; $1170(2): 137-42$.

27. KABIR Y, IDE T. Activity of hepatic fatty acid oxidation enzymes in rats fed alpha- linolenic acid. Biochim Biophys Acta 1996; 1304(2) : 105-19.

28. IDE T, MURATA M, SUGANO M. Stimulation of the activities of hepatic fatty acid oxidation enzymes by dietary fat rich in alpha-linolenic acid in rats. / Lipid Res 1996 ; 37(3) : 448-63.

29. BAUDET MF, et al. Effects of three dietary fats on plasma lipids and lipoproteins in fasting and post-prandial humans after a short-term diet. Lipids $1980 ; 15(4)$ : 216-23.

30. MATTSON FH, GRUNDY SM. Comparison of effects of dietary saturated, monounsaturated, and polyunsaturated fatty acids on plasma lipids and lipoproteins in man. J Lipid Res 1985 ; 26(2) : 194-202. 